\title{
Intravenous immunoglobulin treatment in patients with chronic inflammatory demyelinating polyneuropathy: a double blind, placebo controlled study
}

\author{
M Vermeulen, P A van Doorn, A Brand, P F W Strengers, F G I Jennekens, \\ H F M Busch
}

Department of

Neurology,

Academisch Medisch

Centrum, University

of Amsterdam,

Amsterdam

M Vermeulen

Department of Neurology, University

Hospital Dijkzigt,

Rotterdam

P A van Doorn

H F M Busch

Department of

Immunohaematology

and Bloodbank,

University Hospital,

Leiden

A Brand

Central Laboratory of the Netherlands

Red Cross

Bloodtransfusion

Service, Amsterdam

P F W Strengers

Department of

Neurology, University

Hospital, Utrecht, The

Netherlands

F G I Jennekens

Correspondence to:

Dr Vermeulen, Department

of Neurology - H2,

Academisch Medisch

Centrum, Amsterdam, 1105

AZ Amsterdam, The

Netherlands.

Received 29 April and in

revised form 6 August 1992

Accepted 28 August, 1992

\begin{abstract}
Patients with a clinical diagnosis of chronic inflammatory demyelinating polyneuropathy (CIDP) were randomised in a double-blind, placebo-controlled multicentre trial to investigate whether high-dose intravenous immunoglobulin treatment (IVIg) for 5 consecutive days has a beneficial effect. Fifteen patients were randomised to IVIg and 13 to placebo. In the IVIg treatment group 4 patients improved and 3 patients in the placebo group. The degree of improvement of the patients in the IVIg treatment group was no different from the patients in the placebo group. Electrophysiological studies did not show significant differences between the groups. Since a previously performed cross-over trial showed that a selected group of CIDP patients responded better to IVIg than to placebo, it is concluded that we need better criteria to select CIDP patients for treatment with IVIg.
\end{abstract}

(F Neurol Neurosurg Psychiatry 1993;56:36-39)

Chronic inflammatory demyelinating polyneuropathy (CIDP) can be considered as the chronic variety of the Guillain-Barré syndrome (GBS). The main differences are the course and prognosis. ${ }^{1}$ Patients with CIDP respond to treatment with corticosteroids ${ }^{2}$ and to plasma exchange, ${ }^{3}$ which was demonstrated in randomised clinical trials. Several uncontrolled studies claimed that CIDP patients may also respond to high-dose intravenous immunoglobulin treatment (IVIg). ${ }^{4-9}$ These observations were supported by the results of a double-blind placebo controlled cross-over trial in patients with CIDP, who were judged to have responded to treatment with IVIg and who needed repeated IVIg infusions to maintain their improved condition. ${ }^{10}$ In this cross-over study all patients deteriorated after discontinuation of IVIg treatment. These patients were then randomised to IVIg or placebo (albumin) treatment. Moreover, it was shown that the time lapse from the end of the trial treatment to deterioration was significantly longer after treatment with IVIg than after placebo treatment. Because this cross-over study was car- ried out in a group of selected patients it is still unclear if all newly diagnosed patients judged to have CIDP in whom no other therapy has been started will respond to IVIg. This was investigated in a double-blind randomised placebo controlled multicentre trial.

\section{Methods}

\section{Criteria for eligibility}

Patients eligible for this study were admitted with symptoms and signs of polyneuropathy in the absence of systemic disease, with an electrophysiological diagnosis of demyelinating polyneuropathy based on slowed nerve conduction velocities and or conduction blocks, increased CSF protein (more than $0.5 \mathrm{~g} / 1$ ) and progression of weakness exceeding eight weeks. It was necessary for patients to have a normal erythrocyte sedimentation rate (ESR), haematocrit, white cell and platelet count, serum creatinine, serum glucose, normal liver and thyroid function tests, no antinuclear antibodies, cryoglobulin, or monoclonal protein and also a normal chest radiograph. Patients with a kinship history of neuropathy were excluded as were patients on immunosuppressive treatment. For inclusion in the study all patients required a disability of at least 3 on the modified Rankin scale. ${ }^{11}$ This is a six point scale: $0=$ asymptomatic, $1=$ nondisabling symptoms which do not interfere with lifestyle, 2 - minor disability symptoms, which lead to some restriction of lifestyle, but do not interfere with the patients' capacity to look after themselves, $3=$ moderate disability symptoms which significantly interfere with lifestyle or prevent totally independent existence, 4 = moderately severe disability symptoms which clearly prevent independent existence, but do not require constant attention day and night, $5=$ severely disabled, totally dependent, requiring constant attention day and night.

\section{Randomisation}

When a patient was eligible and after informed consent, the Central Laboratory of the Netherlands Red Cross Blood Transfusion Service Amsterdam (CLB) was informed. The CLB supplied either bottles with immunoglobulin or placebo for a complete treatment course, 
Table 1 Characteristics of the study groups at entry

\begin{tabular}{lll}
\hline & $\begin{array}{l}I V I g \\
n=15\end{array}$ & $\begin{array}{l}\text { Placebo } \\
n=13\end{array}$ \\
\hline Female & 4 & 4 \\
Male & 11 & 9 \\
Age (mean) & 45 years & 50 years \\
Disease duration before entry (mean) & 1 year 2 months & 1 year 9 months \\
No of patients with a remitting course before entry & 2 & 5 \\
CSF protein g/l (mean) & $1 \cdot 16$ & $1 \cdot 62$ \\
CSF cell count/mm (mean) & 5 & 5 \\
NCV peroneal nerve m/s (mean) & 21 & 20 \\
CMAP peroneal nerve mV (mean) & $1 \cdot 5$ & $1 \cdot 9$ \\
NCV median nerve m/s (mean) & 38 & 38 \\
CMAP median nerve mV (mean) & $8 \cdot 5$ & $7 \cdot 5$ \\
Rankin Scale (median) & 3 & 3 \\
MRC Sum Score (median) & 52 & 48 \\
\hline
\end{tabular}

differences not significant

$\mathrm{NCV}=$ nerve conduction velocity in metres per second.

CMAP = compound muscle action potential after distal stimulation of the nerve.

according to a list based on a random number table, to the centre where the patient was admitted. Contents, size and labels of the bottles were not distinguishable. The trial code was broken after the results of all patients had been recorded.

\section{Treatment}

The treatment dose was $0.4 \mathrm{~g} / \mathrm{kg}$ bodyweight/ day for 5 consecutive days and was administered intravenously. The total dose of freeze-dried IVIg was divided into $50 \mathrm{ml}$ bottles, each containing 3 grams immunoglobulin which was dissolved in water immediately before infusion. If a patient was allocated to the placebo group, the same number of $50 \mathrm{ml}$ bottles was given, each

$\begin{array}{lc}\text { IVIg } & \text { Placebo } \\ \mathrm{n}=15 & \mathrm{n}=13\end{array}$

\section{0}

\section{9}

8

7

6

5

4

3

2

1

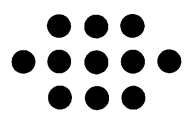

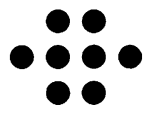

$-1$

$-2$

$-3$ containing 3 grams freeze-dried albumin. The daily dose was subsequently infused within 2 hours. The immunoglobulin concentrate, prepared by the CLB is derived from a plasma pool of more than 3000 voluntary, nonremunerated Dutch blood donors by the cold ethanol cryoprecipitation method (Cohn fraction II). The resulting product was then treated at $\mathrm{pH} 4$ with traces of pepsin to make it suitable for IV use. Its characteristics are similar to those of the immunoglobulin described by Skvaril. ${ }^{12}$ It contains $99 \%$ IgG, $1 \%$ IgA and traces of IgM (total protein content 60 $\mathrm{g} / \mathrm{l})$. IgG subclasses are distributed as follows: IgG1 $57.5 \%$, IgG2 238\%, IgG3 9\%, IgG4 $5.5 \%$. At least $95 \%$ of the IgG is monomeric, less than $7 \%$ dimeric and less than $3 \%$ polymeric. Placebo was prepared from $20 \%$ albumin solution in which less than $0 \cdot 1 \% \operatorname{IgG}$ could be detected. After pasteurisation (10 hours at $68^{\circ} \mathrm{C}$ ) the albumin solution was diluted to $4 \%$ and freeze-dried. All patients who had not responded to the trial treatment received subsequently IVIg for 5 days (open trial) at day 16-21 after the start of the blind trial.

\section{Assessment of treatment response}

At the first day of infusion the disability of the patients was assessed according to the six point Rankin scale. In addition, weakness of three arm and three leg muscles on both sides was assessed using the MRC scale. The following muscles were examined: deltoid, biceps, wrist extensors, iliopsoas, quadriceps femoris and tibialis anterior. The total score (MRC sum score) varies between 0 and 60 points. For assessment of neurophysiological changes; the peroneal and median nerve conduction was measured with surface electrodes on the right extensor digitorum brevis and the right abductor pollicis brevis muscle after supramaximal stimulation of the ankle and knee or the wrist and elbow. The compound muscle action potentials (CMAP) were recorded after distal and proximal stimulation. These assessments and measurements were repeated once between day 16 and 21 after the trial treatment.

Improvement after trial treatment was defined as at least a one point decrease on the Rankin scale and no improvement as unchanged or increased scoring on the Rankin scale. The same neurologist did the first and the last evaluation.

We aimed at randomising 28 patients. This number of patients was based on the assumption that at least half of the patients in the treatment group would improve and none in the placebo group. The ethics committees of the participating centres approved the study protocol. Differences between the groups were analysed with Fisher's exact probability test.

\section{Results}

Twenty eight patients who fulfilled the clinical, physiological and CSF criteria for the diagnosis CIDP $^{13}$ were entered into the trial; 15 were treated with IVIg and 13 with placebo.
Changes in MRC sum scores after IVIg or placebo treatment. The MRC sum scores were assessed before and after treatment and the differences are shown. The MRC sum score is the sum of the MRC score of three arm and three leg muscles on both sides. One muscle score 0-5; sum score 0-60. 
Table 2 Electrophysiological results before and after treatment

\begin{tabular}{|c|c|c|c|c|}
\hline & \multicolumn{2}{|c|}{ Peroneal nerve } & \multicolumn{2}{|l|}{ Median nerve } \\
\hline & $\begin{array}{l}\operatorname{mean} N C V \\
(\mathrm{~m} / \mathrm{s})^{*}\end{array}$ & $\begin{array}{l}\text { mean amplitude } \\
\text { ratio }\end{array}$ & $\begin{array}{l}\text { mean } N C V \\
(\mathrm{~m} / \mathrm{s})^{*}\end{array}$ & $\begin{array}{l}\text { mean amplitude } \\
\text { ratio }\end{array}$ \\
\hline $\begin{array}{l}\text { Before IVIg } \\
\text { After IVIg } \\
\text { Before Placebo } \\
\text { After Placebo }\end{array}$ & $\begin{array}{l}21 \\
24 \\
20 \\
18\end{array}$ & $\begin{array}{l}0 \cdot 73 \\
0 \cdot 71 \\
0.54 \\
0.45\end{array}$ & $\begin{array}{l}38 \\
41 \\
38 \\
36\end{array}$ & $\begin{array}{l}0 \cdot 68 \\
0 \cdot 74 \\
0 \cdot 64 \\
0 \cdot 64\end{array}$ \\
\hline
\end{tabular}

* NCV-nerve conduction velocity (in metres per second).

$\star \star$ Ratio of compound muscle action potential after proximal (knee or elbow and distal (ankle or wrist) stimulation of the nerve (in milli volts)

differences not significant.
The characteristics of the study groups are shown in table 1.

The number of patients improving at least one point on the Rankin scale was similar between the two treatment groups; 4 of 15 patients in the IVIg treatment group improved and 3 of 13 patients in the placebo group. In the treatment group 2 patients improved 2 points in the Rankin score and 2 patients 1 point. In the placebo group all three patients improved 1 point. None of the patients deteriorated in the Rankin score. In the subgroup of patients without a remitting course before entry to the study, 3 of 13 patients treated with IVIg improved compared with 1 of 8 patients in the placebo group. The degree of improvement on the MRC sum score was similar between the groups (figure). Electrophysiological studies showed some differences but these were not significant (table 2).

All patients who did not improve after trial treatment were treated with IVIg (open trial). Of the 10 patients in the placebo group who did not improve initially, 6 had a beneficial response after subsequent $\mathrm{IVIg}$ treatment. None of the 11 patients in the IVIg group who had not responded to trial treatment improved after IVIg treatment in the open trial.

\section{Discussion}

In this study in newly diagnosed patients with CIDP who had no other therapy, we could not demonstrate a beneficial effect of IVIg treatment. This lack of beneficial effect cannot be explained by the method of measuring treatment effect. Improvement was defined as at least a one point decrease on the Rankin scale which is a clinically important improvement. It might be that smaller improvements were not detected by using this scale. Using a more sensitive scale, the MRC sum score, we were also unable to detect differences between the groups.

The interobserver agreement of both the Rankin scale and the MRC sum score has been investigated and appeared to be good. ${ }^{1014}$ Moreover, the same neurologist did the first and last evaluation. Furthermore, a different method, electrophysiological studies, could not detect differences between the groups.

The assessment of treatment response was not carried out too late as none of the patients had initially improved and had deteriorated just before the assessment of treatment response.

A surprising finding was that rapid and dramatic improvement occurred within days after trial treatment in some patients of the placebo group. These patients had a slowly progressive neuropathy of 4 to 6 months duration and after administration of placebo, improvement followed at a rate far faster than the rate of deterioration before the trial treatment. All three patients recovered and needed no repeated treatment. The course in these patients was monophasic, with an onset as in CIDP and recovery as in the Guillain-Barré syndrome. A similar course has recently been described in another report. ${ }^{15}$

We do not believe that improvement in these patients can be ascribed to an active compound in the placebo preparation since such an effect was not seen in the cross-over study in CIDP patients. ${ }^{18}$ Spontaneous improvements in CIDP patients have already been described in the 1950s; Austin described the typical course of untreated patients with CIDP in whom the peak of disability was slowly reached after approximately five months. ${ }^{16}$ From a plateau of disability, these patients then gradually improved and Austin commented that this recovery phase period invariably took longer than the onset of the illness. Dramatic and rapid improvements in CIDP patients treated with IVIg were therefore attributed by us to this treatment. Discovering after the trial code had been broken that some patients in the placebo group had improved rapidly and clinically significantly, we even considered an error in the trial treatment administration. Serum samples taken before and after treatment, however, showed that none of the patients treated with placebo had an increase in serum immunoglobulin level. Today we have little experiences of the natural course of CIDP in patients with marked disability as these patients are usually treated with corticosteroids or with plasma exchange. If the patients who were randomised to placebo had not participated in this trial, they would probably have been treated with high-dose prednisone for prolonged periods, as rapid reduction of the prednisone dose is not recommended because this is considered to carry a great risk of breakthrough of the disease. ${ }^{17}$ The spontaneous and rapid improvement of some CIDP patients observed in this study shows that at least some of these patients may be treated too long with a treatment which is not without risks.

This study included only 28 patients, therefore a type II error should be considered. In calculating the required number of patients, we did not consider the possibility of dramatic improvements in the placebo group. Our hypothesis was that patients in the placebo group would have no clinical significant improvements within two weeks and that at least half of the patients in the treatment group would improve. We calculated that 28 patients would be sufficient to test this hypothesis. Furthermore, in previous studies this number of patients was sufficient to demonstrate treatment efficacy in CIDP. ${ }^{23}$

This small study does not exclude effectiveness of IVIg in CIDP, but we may conclude 
that this treatment is less effective than we had presumed. In a previous study we found improvement after IVIg in 32 of 52 patients (62\%; $95 \%$ confidence limits $47-75) .{ }^{18}$ In this study only 4 of 15 patients improved (27\%; 95\% confidence limits 8-55); a lower figure, although not significantly since the $95 \%$ confidence intervals overlap. A similar low figure has recently been found in an open study on IVIg treatment; improvement in strength or functional tasks was demonstrated in only 3 of 15 CIDP patients. ${ }^{9}$

Recently, research criteria for the diagnosis of CIDP have been published. ${ }^{13}$ All our patients fulfilled the clinical, physiological and CSF criteria for the diagnosis of CIDP, but not the pathological features since nerve biopsy was not required for entry into the study and had rarely been carried out. All the patients, therefore, can be classified as probable CIDP. It is unlikely that nerve biopsy would have changed the diagnosis in many of these patients. In an analysis of a group of 52 patients treated with IVIg we found five simple factors that were related to improvement ${ }^{18}$ : progression of weakness until treatment, absence of discrepancy in weakness between arms and legs, disease duration less than one year, areflexia of the arms, and motor NCV of the median nerve less than $80 \%$ of the lower limit of normal. It was calculated that the probability of improvement after IVIg is $93 \%$ if all these factors are present in a patient with a clinical diagnosis of CIDP. In this study 10 patients in the placebo group and only 6 patients in the IVIg group including the 4 responders, fulfilled these 5 criteria. Prospective studies are needed to investigate if these criteria are useful in the selection of patients who may benefit from IVIg treatment.

We thank the following neurologists for referring or randomisation of patients: $P$ J de Jong, $G$ S D van Leersum, S J Mellema, L J M M Mulder, J A L Vanneste, L H Penning de Vries-Bos, C $H$ Polman, $M J$ J Prick, $H$ J Troelstra, $M$ de Visser and $E M$ de Vries-Leenders. This work was supported by grants from the Prinses Beatrix Fonds and Stichting Willem H Kröger. We thank Professor J van Gijn for his help in the analysis of the results.
1 Dyck PJ, Lais AC, Ohta M, et al. Chronic inflammatory polyradiculoneuropathy. Mayo Clin Proc 1975;50: polyradicul

2 Dyck JP, O'Brien PC, Oviatt KF, et al. Prednisone improves chronic inflammatory demyelinating polyradiculoneuropathy more than no treatment. Ann Neurol 1982;11:136-41.

3 Dyck PJ, Daube J, O'Brien P, et al. Plasma exchange in chronic inflammatory demyelinating polyradiculoneuropathy. $N$ Engl $f$ Med 1986;314:461-5.

4 Vermeulen M, Van der Meché FGA, Speelman JD, Weber A, Busch HFM. Plasma and gamma-globulin infusion in chronic inflammatory polyneuropathy. $f$ Neurol $\mathrm{Sci}$ 1985;70:317-26.

5 Albama M, McNamara ME, Sokol M, Wijschock E. Improvement of neurologic function in chronic inflammatory demyelinating polyradiculoneuropathy following matory demyelinating polyradiculoneuropathy following intravenous gam

6 Cook JD, Delgrado MR, Soutter-Glass D. Treatment of childhood autoimmune polyneuropathy: IV gamma globulin. Neurology 1987;37 (suppl 1):253.

7 Curro Dossi B, Tezzon F. High-dose intravenous gammaglobulin for chronic inflammatory demyelinating polyneuropathy. Ital $\mathcal{F}$ Neurol Sci 1987;8:321-6.

8 Faed JM, Day B, Pollock M, et al. High-dose intravenous human immunoglobulin in chronic inflammatory demyelinating polyneuropathy. Neurology 1989;39:422-5.

9 Cornblath DR, Chaudkry V, Griffin JM. Treatment of chronic inflammatory demyelating polyneuropathy with intravenous immunoglobulin. Ann Neurol 1992; 30:104-6.

10 Van Doorn PA, Brand A, Strengers PFW, Meulstee I Vermeulen $M$. High-dose intravenous immunoglobulin treatment in chronic inflammatory demyelinating polytreatment in chronic inflammatory demyelinating polyneuropathy: a double blind placebo-c
study. Neurology 1990;40:209-12.

11 Van Swieten JC, Koudstaal PJ, Visser MC, Schouten HJA, Van Gijn J. Interobserver agreement for assessment of handicap in stroke patients Stroke 1988;19:604-7.

12 Skaveril F. Qualitative and quantitative aspects of IgG subclasses in IV immunoglobulin preparations. In: Nydegger UE, ed. Immunochemotherapy: a guide to immunoglobuline prophylaxis and therapy. London; Academic Press, 1982:113-22.

13 The ad hoc committee of the American Academy of Neurology AIDS Task Force. Research criteria for diagnosis of chronic inflammatory demyelinating polyneuropnosis of chronic inflammatory demyelinating

14 Kleyweg RP, Van der Meché FGA, Schmitz PIM. Interobserver agreement in the assessment of muscle strength and functional abilities in Guillain-Barre syndrome. Muscle and Nerve 1991;14:1103-9.

15 Hughes R, Sanders E, Hall S, Atkinson P, Colchester A, Payan P. Subacute idiopathic demyelinating polyradiculoneuropathy. Arch Neurol 1992;49:612-16.

16 Austin JH. Recurrent polyneuropathies and their corticosteroid treatment. With five-year observations of a placebocontrolled case treated with corticotrophin, cortisone and prednisone. Brain 1958;81:157-92

17 Dalakas MC, Engel WK. Chronic relapsing (dysimmune) polyneuropathy: pathogenesis and treatment. Ann Neurol 1981;9:134-5.

18 Van Doorn PA, Vermeulen M, Brand A, Mulder PGH Busch HFM. Intravenous immunoglobulin treatment in patients with chronic inflammatory demyelinating polyeuropathy. Clinical and laboratory characteristics associated with improvement. Arch Neurol 1991;48:217-20. 